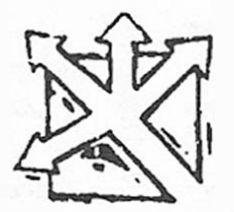

http://doi.org/10.15359/ree.2004-6.13

Educación Superior

\title{
EXPRESIÓN DE LA SEXUALIDAD E INTELIGENCIA EMOCIONAL: UNA EXPERIENCIA EDUCATIVA PARA REFLEXIONAR
}

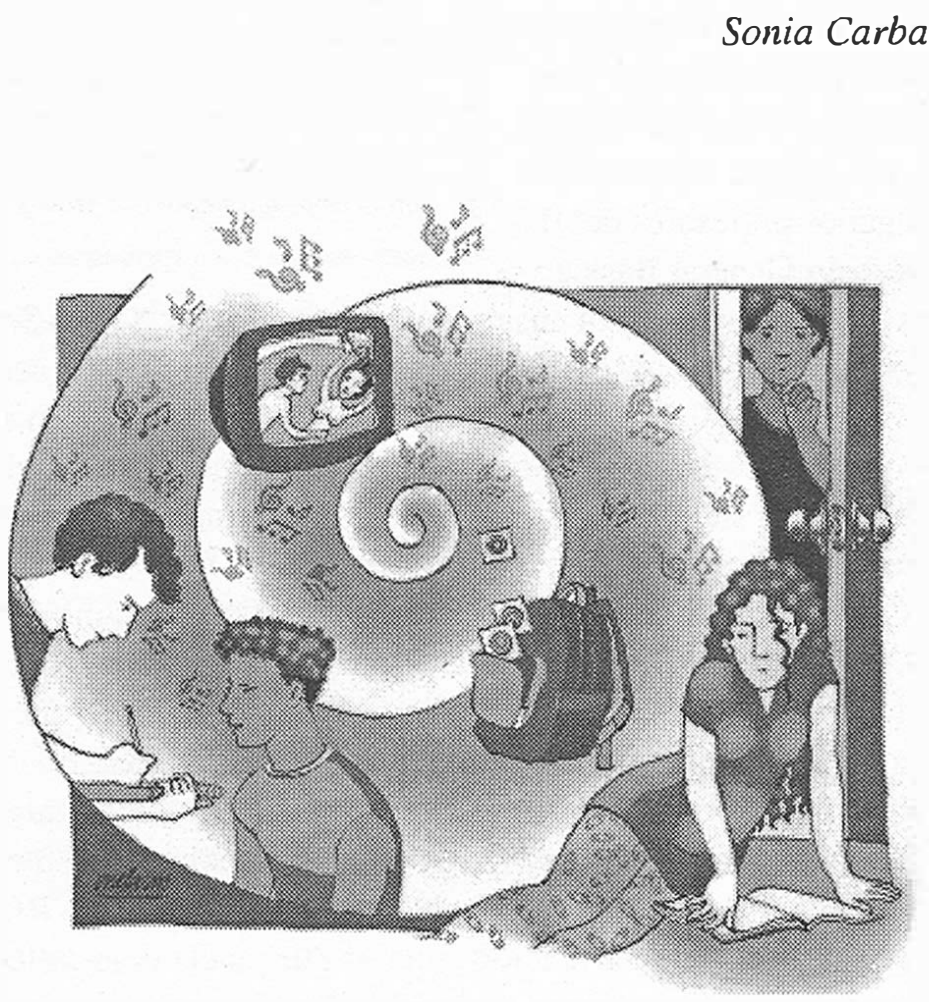

1 Profesora Catedrática de la Facultad de Educación de la Universidad de Costa Rica. Fue profesora de Psicología en instituciones de educación secundaria y es coordinadora de la carrera de Enseñanza de la Psicología en la Escuela de Formación Docente. Durante treinta años ha sido profesora en dicha institución y ha impartido cursos de psicología educativa, desarrollo del niño en edad preescolar y educación sexual, entre otros. Asumió la dirección de la Escuela de Formación Docente de 1996 al año 2000. Dentro de sus últimos artículos publicados están "La lección: su creación"; "Intervenciones para ayudar a construir la inteligencia emocional"; "Retos del maestro y de la maestra del siglo XXI"; y "Educación de expresión de la sexualidad humana". 
"Tí y yo senemos una relación que aprecio y deseo conservar; sin embargo.

cada uno de nosomos es una persona individual con sus necesidades inicas y el derecho de prupar de satisfacer dichas necesidades".

Thomas Gordon

El 12 de mayo de 1992 imparto cl primer "Taller de la Educación de la Sexualidad", como Acción Social, después de más de 15 años de tencr bajo mi responsabilidad cl curso de Educación Sexual en la Facultad de Educación de la Universidad de Costa Rica. Dicho taller responde a la necesidad de educación sexual que expresaron algunos profesores del III ciclo de Educación Gencral Básica y

piofesionales en Orientación cuya misión era trabajar con las "Guías Sexuales" elaboradas por el Centro Nacional de Didáctica del Ministerio de Educación Pública.

La Universidad de Costa Rica como institución de educación superior tiene un compromiso social con los y las costarricenses que se evidencia en los tres pilares que enmarcan su quehacer: investigación, docencia y acción social.

La acción social que realizamos los profesores y las profesoras de la Universidad de Costa Rica nos permite incursionar en diferentes ámbitos del quehacer nacional y comprometemos a aportar elementos para el mejoramicnto de la calidad de vida de los costarricenses. Es desde esta perspectiva que sc gestan, a partir del año 2000, estos talleres de la educación de la scxualidad integrados con la inteligencia emocional, como componente fundamental para expresar la sexualidad humana de una forma cognitiva y emocional inteligente.

Durante cl scgundo ciclo lectivo de la Universidad de Costa Rica, en el año 2002, sc of rece al Centro de Formación Juvenil Zurquí el curso-taller, de Acción Social, "Educación de la cxpresión de la sexualidad e inteligencia cmocional", el cual es muy bicn acogido. El 19 de setiembre de ese año se trabaja la primcra sesión de este taller en dicho centro.

Este curso-taller de "Educación de la expresión de la sexualidad e inteligencia emocional" se desarrolla dentro de un ambiente democrático que permite a las personas confrontar e integrar los conocimientos ya adquiridos con otros nuevos sobre sexualidad humana y cultivar sus habilidades emocionales en la 
The experience described here is based on a workshop about expressing sexuality and emotional intelligence. The course was held at the Corazon de Maria School, located in she Penitensiary Center for young men.

The challenge to free the constructive power that lies within each person is possible only through a type of education that allows him to admit the truth that can be encounter in the ideas of others, 10 create theirown ideas and to have their own criterio that will help them construct and deconstruct any assumprion and behave accordingly. The educational process has not eneled, and we have a lot of hope for these adolescents. They are very similar to the adolescents of our own families. but it is necessany to remember that their personalities and the opportmities they have had in life are unicine; thus, they cannor be seen just like any other seenager we meet every day. convivencia, al expresar y defender posiciones antagónicas o al compartir puntos de vista similares.

Se impartio en la Escuela Corazón de María, ubicada dentro del Centro Penitenciario, durante 8 sesiones de 3 horas cada una y enrumb6 al logro de los siguientes cuatro objetivos generales de la educación de la sexualidad e inteligencia emocional (Carballo, 2002) que se ajustaron al diagnóstico previo que realice antes de iniciar los procesos de enseñanza y aprendizaje:

- Generar una actitud libre de mitos, prejuicios y tabúes hacia la sexualidad humana.

- Construir formas inteligentes cognitiva y emocionalmente de expresar la sexualidad dentro del amor.

- Analizar conocimientos pertinentes y actualizados que contribuyan a la adopción de un código ético, que respalde la expresión de la sexualidad dentro del marco de los derechos humanos.

- Asumir el disfrute pleno y voluntario de la sexualidad sin romper la egosintonia y con protección médica.

Los participantes son 12 adolescentes y una adolescente, cuyas edades oscilan entre los 14 y los 17 años de edad. Están recluidos en el Centro de Formación Juvenil Zurqui, y fueron sentenciados a penas de privación de libertad por la comisión de diferentes tipos de delitos tales como violaciones, asesinatos, robos, entre otros.

Durante la adolescencia el desarrollo evolutivo va alejando a las personas de la niñez y los cambios físicos acelerados de su cuerpo afectan todo su ser. La rapidez del crecimiento corporal y la madurez genital señalan su transformación de niños y niñas en adolescentes y les plantea inquietudes por aceptar y aprovechar sus cambios corporales, comunicarse efectivamente con los demás, fortalecer su papel masculino o femenino y prepararse para un convivencia en pareja. Como es de esperar, la sexualidad que impregna desde la concepción toda su persona, que nace y evoluciona con ella, les produce ahora a estos adolescentes y a esta adolescente nuevas necesidades y despierta deseos y sensaciones que los inquietan. 
Los principios orientadores para esta educación de la expresión de la sexualidad e inteligencia emocional se concretan en:

- Centrar los procesos de enseñanza y de aprendizaje en las caracteristicas y necesidades biologicas, sociales y espirituales de las personas participantes.

- Propiciar la vivencia de un proceso de participación, discemimiento y diálogo que permita a las personas analizar sus mitos, prejuicios, tabúes, conocimientos, actitudes y valores en tomo a la sexualidad humana.

- Facilitar la búsqueda, procesamiento y almacenaje eficiente de investigaciones científicas, con miras al logro de aprendizajes significativos.

- Mediar para que las personas construyan habilidades cognitivas y habilidades emocionales que les permitan expresar, en forma inteligente, su sexualidad.

- Promover un proceso altamente comunicativo que permita la expresión de la diversidad y la autenticidad de cada persona.

- Contextualizar el derecho de los seres humanos a asumir el disfrute libre y responsable de la sexualidad dentro del amor, guiado por sus principios y valores, dentro de un marco de derechos humanos.

Educar a las personas en la expresión inteligente de su sexualidad, es un proceso arduo y placentero, dinámico y nunca acabado que contribuye a que la persona se acepte a sí misma en la diaria convivencia humana, que acepte y respete a los otros seres humanos y a que se ajuste responsablemente a los avances y cambios de los tiempos.

Al educar la expresión de la sexualidad y la inteligencia emocional de estos adolescentes y de esta adolescente el gran reto que guía el quehacer de cada sesión es formarlos como seres autónomos, capaces de pensar por sí mismos, de criticar, de expresar su propia opinión de tal forma que puedan diferir o compartir posiciones y actuar de manera inteligente emocional e intelectualmente. De acuerdo con Kamii y Devries (1971) los tres principios de la enseñanza que se deben considerar cuando se aboga por la autonomía como finalidad de la educación son:

- Reducir el poder del profesor como adulto que es, en cuanto le sea posible, e intercambiar sus puntos de vista con los y las jovenes en una relación equitativa. La relación jerárquica y unilateral obstruye en la persona la construcción de sus propias ideas y valores morales.

- Es esencial que el profesor aliente a los jovenes y las jovenes a intercambiar y coordinar puntos de vista entre ellos. 
- Es fundamental que el profesor estimule a los adolescentes y a las adolescentes a ser mentalmente activos (por ejemplo, a ser curiosos, a tener iniciativa, a ser críticos y a relacionarse con las cosas) y a tener confianza en su propia habilidad para descifrar los problemas.

La fe en que es posible fortalecer factores de resiliencia en el ser humano y la crecncia en procesos capaces de contribuir a que las personas se sobrepongan a la adversidad hacen que estc curso-taller de Expresión de la sexualidad e inteligencia emocional, oriente sus estrategias pedagógicas a promover en los participantes el fortalecimiento de la autoestima, la asertividad, la empatia y la congruencia, en un marco de relaciones personales que les dé seguridad y apoyo para practicar las habilidades de autoconocimiento, automotivación, autocontrol, empatía y relaciones sociales.

A continuación se presentan una serie de anécdotas que reflejan algunas de las vivencias del curso-taller con orden y secuencia, las cuales se escriben horas después de concluida la sesión del dia y constituyen verdaderas reflexiones evaluativas. Estos momentos seleccionados para redactar este artículo, son reconstruidos y planteados desde una posición más académica y menos emotiva de los que se conservan en el cuaderno de apuntes. Todos ellos proyectan una educación de la expresión de la sexualidad e inteligencia emocional en la relación de la persona consigo misma y en la convivencia con las otras, a través de los vínculos emocionales, del papel sexual, de la respuesta sexual y el erotismo y de la reproducción.

La primera vez que visité el Centro de Formación Juvenil Zurquí fui recibida muy amablemente por al señora Directora de la escuela y al expresarle mi preocupación por el retraso que sufrí, ella muy amablemente me dijo: "aquí nadie se preocupa por el tiempo, pase adelante y bienvenida". Inmediatamente mandó a traer a los jóvenes y a la joven, a la escuela que está dentro del complejo penitenciario. Fue todo un despliegue de seguridad. Cada joven venía esposado y al llegar a la escuela se le quitaban las esposas. Este detalle para mí fue impactante y a él se sumó el hecho de que en el aula permanecieran dos personas de seguridad, bien armadas, una en la puerta y otra en la parte de atrás de aula.

El aula era amplia, limpia y los pupitres estaban perfectamente acomodados. Los jóvenes y la joven se acomodaron en los pupitres, conversaban entre ellos y el murmullo de voces llen 6 el aula. La señora directora me present 6 a las personas y en pocas palabras les saludé, les expresé los objetivos del cursotaller y mi gusto por estar allí, omitiendo comentarles lo impactada que estaba por la "seguridad" en el aula. 
Durante esta primera scsión del curso-taller los y la adolescente reciben, como estrategia pedagógica, hojas de colores en las cuales escriben las siguientcs inquictudes y neccsidades relacionados con la cducación de la expresión de su scxualidad:

- "Las consecuencias de las relaciones sexuales".

- "Si teniendo una relación de parcja, cl sexo se debería de controlar para no hacer un hábito".

- "Sobre sexo oral".

- "Me gustaría saber todo sobre sexualidad".

- "Quisiera saber, ¿ por qué los condones no son tan efectivos en una relación?"

- "Yo quicro saber sobre la sexualidad lo más importante, por cjemplo, sobre la convivencia".

- "Si el sexo anal es correcto".

- "La mujer siente el mismo placer cuando ticne relaciones con un hombre de pene pequeño o grande".

- "La comunicación con las parejas... ¿por qué a veces no se entienden hablando?".

- "Una mujer siente más excitación cuando le hacen cl scxo oral".

- "Scxualidad con pareja".

- "Qucría saber todo, ¿cómo romper un "virgo"?.. debe ser rico".

- “Si uno se soba se debilita?".

- "Sobrc sexualidad".

- "Sobre sexo oral".

Terminada esta tarea, recogí las hojas y escuché los comentarios jocosos sobre la temática quc hicieron los jóvenes.

Estos mensajes son básicos para diagnosticar el nivel de conocimiento que se debc cubrir en la programación de las sesiones, durante las cuales las interacciones abicrtas entre sus participantes propiciarán el diálogo para fortalecer las habilidades de la inteligencia emocional.

En realidad cstaba agotada emocionalmente y los comentarios que hicc fueron pocos, pensé que cra prudente espcrar para aportar y guiar la discusión y cl diálogo.

Los invile a sentarse en posición cómoda frente a la tclcvisión y les proyecté un documental sobre adelantos científicos. La cinta cautivó a los presentes y al terminar pedí comentarios. Tres personas comenzaron a la vez a comentar y a preguntar. Lcs pedí silencio orden, y respeto para el compañero o compañica que desee participar y asi lo hicieron. Las preguntas y comentarios giraron sobre 
las temáticas de la clonación, cuántos genes tenemos, si sc congelan seres humanos enfermos para revivirlos después de que se descubra la cura para su padecimiento. Con cierta timidez reforce y amplie comentarios y así surgieron nuevos interrogantes que plantearon cl costo económico de las investigaciones y lo difícil que es para nosotros los costarricenses tener acceso a esos avances tecnológicos y científicos en este momento.

Me despedí y algunos muchachos y la muchacha se acercaron a mí y me pidieron que no cnseñara a nadic del Centro lo que ellos y ella cscribieron en las hojas que me llevaba.

En la noche de cse dia revise el trabajo rcalizado y conforme lo analizaba mi alma empezó a cncontrar paz. Estos muchachos y esta muchacha cxpresaban las mismas dudas, inquietudes e ignorancias sobre expresión de la scxualidad humana, que los jóvenes y las jóvencs de las instituciones de educación secundaria que yo visitaba.

En la segunda sesión, tomando en cuenta los incereses y necesidades de estos jóvenes y de esta joven, se clarifican con ellos y ella los términos expresión de la sexualidad e inteligencia emocional, partiendo de que el ser humano tiene múltiples inteligencias y que como ser único necesita de la educación para expresar su scxualidad en forma intcligente intelectual y emocionalmente en los vínculos emocionales, en los papeles sexualcs, en la respuesta sexual y crotismo y en la reproducción.

En la pizarra se dibuja un triángulo y se inicia el diálogo sobrc la tríada de la salud, cnfatizando la importancia de la egosintonía, la protección médica y del respeto a los dercchos humanos en la expresión voluntaria de la sexualidad. Se establece una conversación muy amena cuando cada pcrsona expresa cómo le dice a la respuesta sexual y se rcscata la importancia de que se use cl vocabulario adecuado al interactuar en estc grupo. La gran pregunta que cerró esta sesión la formuló uno de los muchachos: “¿Puede el adolescente tener respuesta sexual?”. Se les plantc $\delta$ a los presentes que quedaba pendicnte reflexionar al respecto, pucs con ese cuestionamiento se iniciaría la siguientc jornada de trabajo.

En la educación en la expresión de la sexualidad humana es palpable, a cada instante, el enlace inseparable entre razón y emoción. Para iniciar la tercera sesión se les muestra a los muchachos y a la muchacha un documental tomado de la televisión sobre las hormonas en la adolescencia, en el cual, de una forma maravillosa, se plantea el desarrollo de los adolescentes y de la adolescente y se enfatiza cómo el desarrollo físico se acelcra, dejando atrás al desarrollo cognitivo y emocional. Después de ver dicho documental se analiza la expresión de la sexualidad en la respuesta sexual, en la adolescencia, a la luz de la tríada de la salud. 
El primer comentario que expresa en voz alta un joven es: "Todo está muy claro, sí podemos tener respuesta sexual, pero a la vez no podemos. Entonces, ¿cómo es el asunto?" Se les explica que la expresión de la sexualidad en la respuesta sexual es una opción que tienen los hombres y las mujeres durante la adolescencia, ya que sus cuerpos están casi listos físicamente y las hormonas les envían sus mensajes motivadores para ello, pero que desde el punto de vista de la egosintonía cada uno y cada una debe analizar si la vive o no voluntariamente. Se les aclara y reafirma a estos jóvenes y a esta joven que egosintonía implica estar en paz y armonía con uno mismo o con una misma, por lo que es importante comportarse de tal forma que se respete nuestra escala de valores y los derechos humanos. Asimismo se les recuerda tener presente la protección médica para no contraer infecciones de transmisión sexual y para evitar un embarazo no deseado.

Esta sesión del curso-taller se cierra solicitando a los jóvenes y a la joven que en una hoja respondan las siguientes preguntas: ¿Qué aprendieron? ¿Cuáles aspectos de expresión de la sexualidad desean conocer o profundizar? Seguidamente se transcriben las respuestas que estas personas dan a esas preguntas:

- "Me gustaría saber sobre el placer sexual de la mujer; sexo oral. Me gustan las lecciones. Todo está claro".

- "Yo he aprendido sobre sexualidad cosas interesantes y quiero saber sobre el SIDA".

- "Que el hombre y la mujer tienen una etapa distinta en la sexualidad. Me gustaría que diera más cursos de sexualidad".

- "Que el hombre y la mujer tienen una etapa distinta".

- "Aprendí sobre la pubertad, sobre los orgasmos de la mujer y de los hombres. Me gusta todo lo aprendido. Quiero saber más".

- "Yo quiero saber más del sexo. Porque ya soy adolescente y me gustaría saber por qué las mujeres son tan bonitas y tan atractivas y tan bellas".

- "Bueno yo aprendí sobre la expresión de la sexualidad. Me ha ayudado ha comprender que el sexo no es algo para estar jugando, es algo en verdad para expresar sentimientos".

- "Aprendí a saber unas de las partes del cuerpo de la mujer y del hombre, el crecimiento de las partes del ser humano. Aprendí a respetar las opiniones de mi pareja en el acto sexual. También a saber a qué se refiere el sexo. Quisiera aprender más y más del ser humano".

- "Aprendí que el sexo no es un juego, que hay que cuidarse. Que en la cama se comienza una relación". 
- "Yo quiero saber más acerca de los hombres y mujeres y también acerca del sexo y otras cosas. Aprender más sobre todo me interesa".

- "Muchas cosas importantes".

- "Me gustaría seguir aprendiendo sobre sexualidad. He aprendido muchas cosas, lodo me interesa".

- "He aprendido a entender que los hombres y mujeres no solo son sexualidad sino que también hay sentimientos y responsabilidades tanto en una relacion de pareja como en lo personal... y del curso en sí opino que es muy agradable para mí y me gusta saber cómo aceptar a mi pareja tal como es".

- "Yo he aprendido acerca de los orgasmos fcmeninos y masculinos y sobre el desarrollo del cuerpo humano y quisiera seguir aprendiendo mucho más acerca de la sexualidad".

Esta evaluación me satisface y siento que el esfuerzo valió la pena. Los jovenes y la joven expresan con claridad no sólo los logros sino también el deseo de profundizar en el conocimiento de la expresión de la sexualidad humana. Por lo tanto el camino a seguir en las siguientes sesiones se vislumbra prometedor, con grandes retos pedagógicos para dar contención a las expectativas de los muchachos y de la muchacha.

La sesión número cuatro se estructura con la estrategia pedagógica conocida como análisis de casos. Durante la puesta en común del trabajo de los cuatro grupos de tres personas que se forman, se siente lo escueto y profundo de las reflexiones que se plantean. La fluidez de palabra y la expresión de sentimientos en forma oral, ante las otras personas, es un permiso que estos jóvenes y esta joven no se dan. Se limitan a leer el texto del caso que se les dio, a señalar algunos retos en la expresión de su propia sexualidad y por supuesto, se vislumbra el temor al futuro. Seguidamente se presenta a todo el grupo de personas el caso de la pareja "David y Soledad" y se les escribe en la pizarra las siguientes preguntas generadoras: ¿Quiénes de verdad dan afecto y amistad a David y a Soledad? ¿Cuáles son las personas que ayudan a crecer a los y a las adolescentes? ¿Cuáles son las habilidades propias de la inteligencia emocional que se perciben en los personajes?

Para concluir se les pide a los jovenes y a la joven que inventen el final de esta historia de amor entre David y Soledad. A ellos y a ella, les gustó mucho la historia y se percibe en sus expresiones cortas y concisas, la sistematización de conocimientos que les permitirá enriquecer su toma de decisiones.

En la quinta sesión de este curso-taller se continua con el abordaje pedagogico de la expresión de la sexualidad, siguiendo las inquietudes e intereses expresadas por ellos en la tercera sesión. 
Como estrategia pedagogica se emplea la lluvia de ideas. Se escribe en la pizarra la palabra "amor" y cada persona pasa a escribir una palabra que asocia a ella. La lluvia de palabras entorno al amor se visualiza así:

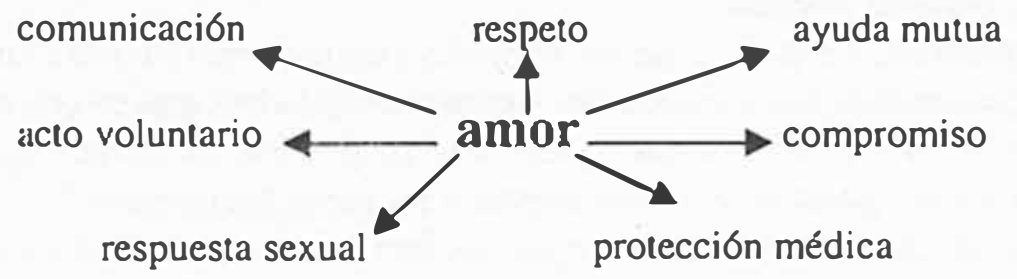

Se dialoga nuevamente sobre las fases de la respuesta sexual tanto femenina como masculina y se refuerzan muchos conceptos en tomo a la expresión de la respuesta sexual con ayuda de ilustraciones de la anatomía y la fisiología del cuerpo humano. Para concluir esta parte de la sesión se escribe en la pizarra la siguiente anécdota: "Marta de 16 años, tuvo respuesta sexual con Jaime de 17 años. Maıta llega y me cuenta a mí: No sentí nada, se lo dije a él y solt6 una carcajada".

La muchacha y los muchachos leen, con cierto asombro e incómodos algunos, el texto escrito y cuando se solicitan comentarios al respecto se escuchan unos murmullos, pero en realidad no desean decir nada en voz alta. Ante tanto silencio no esperado, se les refleja su comportamiento y se les solicita "permiso" para comentar la situación de Jaime y Marta. Es entonces que surgen escuetas afirmaciones: "Jaime está herido en su masculinidad". Se aprovecha para puntualizar que en nuestra cultura a Jaime se le responsabiliza del orgasmo femenino y se piensa en él como el príncipe azul que tiene que saber todo sobre el disfrute sexual. Inmediatamente un joven exclama: "Así tiene que ser". Se les estimula a reflexionar sobre los derechos del hombre y de la mujer y sobre la importancia de la educación de la expresión de la sexualidad en la respuesta sexual para que cada uno asuma la responsabilidad que les corresponde en el disfrute pleno y para que mediante la comunicacion se expresen mutuamente cómo vibra él y cómo vibra ella. Se aclara que no siempre la introducción del pene en la vagina produce satisfacción y orgasmos en la mujer y que el juego amoroso previo es fundamental para que con el estímulo de sus zonas erbgenas, la mujertenga orgasmos previos a la introducción del pene en la vagina. Como impulsado por un empujón muy fuerte, un joven dice: “ ¿Pero cuando vamos a tener tiempo y un lugar apropiado para todo eso?" Otro joven pregunta: "En la violación ¿la mujer siente?".

Al retomar el diálogo se puntualiza en la importancia de disponer de tiempo para disfrutar de la respucsta scxual voluntaria, previendo embarazos no deseados 
e infecciones de transmisión sexual. También se replantea el papel del cerebro en el disfrute de pleno de la respuesta sexual y se analiza cómo durante una violación no se dan los principios claves que se puntualizan. Es pertinente indicar que es otro joven el que concluye: "O sea, estamos listos físicamente para tener respuesta sexual, no lo estamos emocionalmente y hay' alrededor nuestro una serie de situaciones que nos niegan los conocimientos, el tiempo y el espacio apropiado". Este comentario se retoma para cerrar la sesión y se explican de nuevo las habilidades de la inteligencia emocional con ejemplos que aportan las personas presentes sobre la importancia de las mismas para el disfrute de la respuesta sexual.

Antes de finalizar la sesión, los jovenes y la joven escriben en una hoja lo que aprendieron y las dudas que aun tienen. Dos muchachos manifiestan que no desean escribir y con voz fuerte dicen: "explique sobre embarazo y parto" y "deseo saber todo sobre la formación del hijo".

En las hojas que entregaron los jóvenes y la joven que sí escribieron, dan los siguientes mensajes:

- "Aprendí que para que la mujer tenga excitación hay que tener un juego amoroso primero y las etapas que hay en la excitación, quisiera saber más de las responsabilidad que hay entre la pareja a la hora de decidir cuándo se vil a tener un hijo".

- "A mí me gustaría seguir aprendiendo más cosas sobre el amor".

- "Bueno, a mi me parece que este curso me ha hecho ver que la respuesta sexual es algo con lo que no se debe jugar porque el placer sexual es algo con lo que no se debe jugar".

- "Yo aprendí cómo se hace el amor, quisiera aprender mucho más del sexo y quisiera aprender sobre la eyaculación".

- "Hoy aprendí sobre los cambios físicos de la mujer y cómo hacer una mejor respuesta sexual y los cambios de la vagina y quisiera saber más sobre la eyaculación de la mujer".

- "Yo aprendí con mucho respeto si las posiciones a la hora de tener acto sexual son agradables o ayudan a tener una mejor complacencia sexual".

Estos mensajes son una retroalimentación evaluativa que determinan pautas para reorganizar las sesiones que faltan. El éxito de la educación de la expresión de la sexualidad reside en ajustarla siempre a los avances de aprendizaje de los y la participantes y a la discusión y aclaración de las nuevas dudas e inquietudes que surjan. Plantear y replantear los conocimientos y las posiciones personales sobre expresión de la sexualidad es una labor trascendental para construir 
formas de coniportamiento que enriquezcan la inteligencia emocional de cada ser humano en convivencia con los demás.

Lo fundamental en la sesión número seis es trabajar la relación de pareja. Para esto se utiliza como estrategia pedagógica la lectura de un cuento sin incluir el final de la historia. En él se refleja la relación entre los personajes Lolo y Lala. Lolo le declara su amor a Lala y le pide que se case con él. Sin embargo, ella decide irse a "descubrir nuevas latitudes y amistades". Seguidamente los jovenes y la joven deben escribir el final del cuento, pero como no desean escribir, se concretan a decir en voz alta sus finales:

- "Lolo va a la ciudad a buscar a Lala y regresan juntos".

- "Lala regresa, busca a Lolo y se queda con él".

- "Lolo y Lala no pueden vivir el uno sin el otro".

Como es costumbre la joven presente no hace aportes orales de ningún tipo. A los presentes les parece inapropiado el planteamiento que se les hace en el sentido de que Lala se autorrealiza como pianista y que vive feliz lejos de Lolo.

En seguida se les solicita a los jovenes y a la joven que formen grupos de tres y que escriban con un marcador de color, en un papel periódico blanco grande, por un lado ¿qué es ser femenina?, y por otro ¿qué es ser masculino?, en nuestra cultura. Al concluir la tarea se pasa a una reflexión evaluativa sobre lo escrito. Cada uno de los tres carteles construidos por los grupos dicen que ser masculino es:

1. Ser hombre, responsable, educado, respeto, depende de uno mismo, fuerte, tener una mujer, mantenerla, trabajar

2. Ser responsable, fuerte, no llorar, ser serio, trabajar, ser la cabeza del hogar, estudiar, no jugar con muñecas, relacionarse, quererse, tener ciertas labores que realizar diferentes a las mujeres.

3. Ser hombre, tener el poder, cargar con la responsabilidad del hogar, ser fuerte, ser trabajador, no llorar, no ser comelón.

Y sobre ser femenino escriben:

4. Ser mujer, ser ama de casa, débiles, sensibles; para el oficio, para complacer al hombre de noche, para ser mamás.

5. Lloronas, flexibles, débiles, tienen que limpiar el hogar, tienen que cuidar a los niños, son sexys, lindas, coquetas, tienen que obedecer al marido, son 
diferentes a los hombres, tienen que darse a respetar, son las que educan a los hijos.

6. Ser débiles, lloronas, ser responsable con sus hijos, ser ama de casa, trabajadoras, acaparadoras, son comclonas.

Los jóvenes comentan en forma oral los cstereotipos masculinos y los confrontan con los femeninos que ellos poscen, como llorar y ser coquetos. Pero, a pesar de que se afirma que los estcrcotipos sociales impiden el desarrollo pleno dc los scres humanos, dos muchachos expresan que para ellos cl poder del hombre sobre su mujer es un hecho, aún cuando la mujer trabaje fuera del hogar. Los otros muchachos aceptaron la afirmación y la única muchacha del grupo expresa: "A mí no me parece". La atmósfera del grupo se altcra y se genera la energía creadora que produce los antagonismos al enfrentarse. Con rapidez, otro joven dice con firmeza: "Nunca permitiré el cambio de papeles sexuales". Esto anima a otro muchacho a aclarar más la posición machista que tienen estos varones, pues afirmo: "Estoy de acuerdo con la equidad en la relación de parcja, siempre y cuando sea yo cl que mande".

Como comentario a este último mensaje, sc le aclara al grupo la confusión de expresiones que se han dicho y se les anima para que reflexionen y asuman posiciones más acordes con una democracia de género, sin olvidar que el camino a recorrer es arduo y largo.

Al término de la sexta sesión se les muestm un documental tomado de la televisión sobre la historia del sexo en el siglo $X X$, al que atienden con interés y los comentarios finales son, aunque cortos, muy significativos. Sin embargo, se hacen muy rápido porque desean ir a almorzar. Sc siente que realmente el proceso de educación de la sexualidad aporta un grano de arena al conocimiento de éstos seres humanos.

Son lamentables las ausencias de uno u otro muchacho en ciertas sesiones, pero la vida en el Centro es una rutina muy complicada por acontecimientos imprevistos, tales como tráfico de drogas, violaciones, abusos scxuales y fugas de personas internas. Aún así se logra el avance en las sesiones. Tal como me afirmó un día la señora dircctora del Centro: "esto pasa porque estamos en una cárcel", lo cual yo comprendí pcrfectamente.

La sétima seșión se inicia con un cuento que explica cómo hay que vivir sin miedo al futuro. Como estrategia pedagógica, un muchacho lec una parte del cuento y luego otro continúa hasta concluir. Se percibe que el cuento llega al alma de las personas, las cuales resaltan aspectos del cuento que les parecen fabulosos, repitiendo textualmente las palabras del autor. Se les refuerzan sus reflexiones y se les dice cómo en cada instante de la vida se viven situaciones que son todo un reto para el ser humano y ante ellas se debe tomar decisiones. 
Se muestra luego un documental tomado de la televisión que enfoca el desarrollo intrauterino como la primera maravilla, en un leguaje pro vida, que invita a cada hombie y a cada mujer a hacerse responsable del hijo que engendie. El documental conmueve a los muchachos y aún cuando tienen prisa por ir a almorzar, expresan lo siguiente:

- "Yo en el útero de mi mamá, tenía hermano de agua".

- “El sexo lo determina el hombre?".

- "Señora, ¿ducle mucho parir?".

- “QQué se siente tener un niño que va creciendo dentro del útero?”.

- "¿Por qué si duele tanto a las mujeres, tienen más de un hijo?".

- "Nosotros sí vimos un parto de verdad".

Con la premura del momento, estas participaciones de los jovenes son reforzadas reflejando sus palabras. Asimismo, sc les contcstan las preguntas enriqueciéndoles los conocimientos y enfatizando por qué hoy en día sc habla de familia gestantc. Al evaluar el trabajo de la sesion, se hace un recucnto claro de los destellos de las habilidades emocionales que estas personas están proyectando y se siente satisfacción por los logros cognitivos y emocionales en proceso.

La sesión número ocho es una sesión evaluativa final. Se planea como un cierre que "amarre" los conocimientos nuevos y viejos y que les permita a los jovenes y a la joven expresar sus avances en una expresión de la sexualidad cognitiva y emocionalmente inteligente. Se inicia con la prosa poética "Tú y yo" de Thomas Gordon (1978). Esta página inmortal enfatiza las habilidades de autoconocimiento, automotivación, autocontrol y empatía claves para relacionarse con uno mismo o con una misma en las relaciones con las demás personas. En ella cl respeto a los derechos humanos, la comunicación y la negociación constante dentro del marco del amor, dan una hermosa pincelada de manera profunda y sencilla, a la relación emocional inteligente entre dos seres humanos. Al abrirse el espacio para el diálogo grupal se escucha un gran suspiro y frases del texto son repetidas por una u otra persona en voz alta, sin ningún orden, pero con sentimiento profundo.

La evaluación formativa final consiste en darle a cada muchacho y a la muchacha el texto "ser capaz de elegir cuándo y cọn quién", para que con base en él contesten cuatro preguntas que están escritas en la pizarra.

Seguidamente se presentan las preguntas y las respuestas respectivas dadas por las personas: 
1. Explique con sus propias palabras, ¿Cómo vivir su sexualidad de mancra que pueda sentirse protegido y adcmás alcanzar la mayor plenitud sexual?

- "Conocer la historia sexual de la parcja".

- "Tener una sola compañera".

- "Usar preservativo. No usar drogas".

- "Ser capaz de elegir cuándo y con quién tener relaciones sexualcs".

- "Respetar la opinión de cada uno".

- "Tener una pareja, amarla y respetarla".

- "Evitar infecciones de transmisión sexual y embara\%os no deseados".

2. Explique con sus propias palabras, ¿Por qué la expresión de la sexualidad nos compromete a un nivel muy profundo?

- "La scxualidad no es solo el acto físico, abarca la totalidad de nuestro ser".

- "La sexualidad nos compromcte a nivel corporal, emocional y espiritual".

- "Porque genera disfrutc y responsabilidad".

- "Porque debemos cuidarnos de enfcrmedades de transmisión sexual".

- "Porque no se conversa este tema de la sexualidad con cualquicr persona y abarca campos muy intimos.".

3. Explique con sus propias palabras, cuatro condiciones que debe tener en cuenta para alcanzar plenitud sexual y proteger toda su persona.

- "Mantencr un equilibrio entre niveles físicos, emocionales y espirituales en la relación".

- "Comunicarse y asumir compromiso".

- "La fidelidad también ayuda al equilibrio en una buena relación".

- "Amor, respeto, y sobre todo mucho amor".

- "Usar preservativo y no andar de mujer en mujer".

- "Tener una sola pareja".

- "No consumir drogas. Anulan el dominio propio y la capacidad de tomar decisiones".

- "Quererse, respetarse, ser fiel".

- "Conversar mucho, respetarse. Pedir ayuda a una persona mayor".

- "Pasar ticmpo juntos". 
4. Explique con sus propias palabras, cuatro aspectos que una pareja que se ama genuinamente tienc que tener presentes:

- Amor, igualdad, respeto, comprension.

- Comunicación, confianza, amor mutuo.

- Expresar sentimienros.

- Compartir, dcsearse.

- No embarazarse si no se desea.

- Libertad de elegir.

- Protección legal.

- Compromiso mutuo.

Las respuestas que estos jóvenes y esta joven dan están muy apegadas al texto de apoyo y no cumplen a cabalidad lo que les pregunta. Sin embargo, es un avance que seleccionen los conceptos pertinentes para cada pregunta. En casi todos los momentos del curso-taller, se siente que es la primera vez que estos adolescentes y esta adolescente escuchan y dialogan sobre conceptos de sexualidad $e$ inteligencia emocional y es evidente que son pocos los espacios que tienen para este tipo de reflexiones.

A manera de reflexión final cs pertinente retomar el pensamiento de la Dra. Caldwell (s. f.) y aplicarlo a este grupo de personas que participaron en este curso-taller de Educación de la expresión de la sexualidad e inteligencia emocional:

"De alguna manera cada adolescente es como todo los adolescentes. De cierta manera es como algunos y algunas adolescentes y de cierta manera no se parece a ningún adolescente ni a ninguna adolescente".

Al igual que Carl Rogers (1980) se considera que la cducación de los y de las adolescentes tiene la fuerza suficiente para liberar el poder constructivo que existc dentro del organismo humano y que lleva a la persona hacia la realización plena de sus posibilidades. La educación rescata el derecho del ser humano a afirmar su dignidad y al igual que la vida, fluyc como un río, aparentemente sin sentido, nunca con la misma agua, siempre corriendo, sin que nadie sepa qué sucederá è el momento siguientc.

También, se comparte el criterio de Delors (1996) que refuerza la importancia de la educación al afirmar que es la educación durante toda la vida, la que permite una reestructuración continua de la persona, de su conocimiento, de sus actitudes, de su facultad de juicio y acción para responder de manera flexible y diversa a los retos de los tiempos. La educación encierra un tesoro y sus alcances llevan a aprender a vivir juntos, a aprender a conocer, a aprender a hacer y a aprender a ser. 
Hoy dia los investigadores de la sexualidad humana y de la pedagogía heredan a la humanidad una amplia gama de conocimientos, más no se puede olvidar la historia de vida de los y las participantes, quienes tienen que establecer un puente cognitivo entre el cúmulo de sus experiencias y los nuevos aportes que aprenden, y construir significados que les permitan comportamientos inteligentes cognitiva y emocionalmente al expresar su sexualidad.

Por lo tanto, el ¿qué enseñar?, se tiene que integrar al ¿cómo?, y al ¿para qué?, porque no son únicamente los conocimientos lo que requiere el ser humano, sino que se hace necesario reflexionar y analizar los conocimientos a la luz de sus propias actitudes, sentimientos, valores y pensamientos con miras a ajustarlos a la situación sociocultural, psicológica y espiritual de la persona.

Sólo un educador o una educadora que conozca y acepte a plenitud su naturaleza humana de ser sexuado, podrá orientar en forma satisfactoria el proceso educativo de la sexualidad al contribuir con sus actitudes sanas a desterrar los dogmas, las inquisiciones, las ignorancias y los fanatismos de diversa índole acerca de la sexualidad humana. Admitir la verdad que hay en las concepciones ajenas, elaborar las propias y tener criterio independiente requiere de un sentido universal que permita construir y reconstruir posiciones.

Es así, como la preparación académica, psicosocial y espiritual, la habilidad de comunicarse, la creatividad y la intuición de la persona responsable de planear, ejecutar y evaluar los procesos de enseñanza y aprendizaje de la sexualidad es la clave para que la integración del ¿qué?, ¿cómo?, y ¿para qué?, sean un éxito al ajustarse a las necesidades de los participantes.

Los y las adolescentes que se encuentran privados de libertad y sometidos a una serie de limitaciones y carencias, necesitan con urgencia acciones educativas que abran espacios para su desarrollo integral. Los efectos de las privaciones sociales, cconómicas, espirituales y afectivas que ellos sufren son acumulativas y el dolor, la culpa, la rabia y sus preocupaciones en tomo a su vida, son vivencias cotidianas que necesitan contención de equipos interdisciplinarios.

Es importante dar seguimiento a este tipo de esfuerzos para que no se conviertan en un hecho aislado. Hay que tener esperanza en estos seres humanos. Todos ellos son como los y las adolescentes de nuestras familias, sin embargo, son tan únicos en su personalidad y en las oportunidades que les ha brindado la vida, que no se parecen a ninguno de los y las adolescentes que cotidianamente nos rodean.

"Se quedaron allí arriba en silencio un buen rato.

- ¿Cómo podemos sertan distintos y sentimos tan iguales? - meditó Flitter. - ¿Y cómo podemos sentimos tan distintos y ser tan iguales? - se preguntó Pip. 
-Creo que eso es todo un misterio - pió Flap.

-Estoy de acuerdo --dijo Stelaluna-. Pero somos amigos. Y eso sí que está claro".

Janell Cannon (1994)

\section{Referencias}

Cannon, Janell. (1994). Stelaluna. Barcelona: Editorial Juventud.

Carballo, Sonia. (1980) "Carl Rogers: de la psicología a la educación". Educación 4(1), pp. 55-76.

Carballo, Sonia. (1983) "Promover el desarrollo del educando como persona sana". Educación 7(1-2), pp.45-60.

Carballo, Sonia. (1998) "Intervenciones para ayudar a construir la inteligencia emocional". Educación 22(1)pp. 77-89.

Carballo, Sonia. (2002) "Educación de la expresión de la sexualidad humana". Educación 26(1). pp. 29-46.

Delors, Jacques. (1996) "La educación encierra un tesoro". Madrid: Santillana y Ediciones UNESCO.

Goleman, Daniel. (1995) "La inteligencia emocional". México D.F.: Javier Vergara Editor.

Gordon, Thomas. (1978) P.E.T. en acción. México D.F.: DIANA.

Kamii, Constance y Devries, Rheta. (1971) Teoria de Piaget. España: Artez.

Massoni, Reinaldo. (2000) Sexo: energia fundamental para la vida. México D.F.: Editorial Aguilar, Alea, Taurus, Alfaguara S.A.

McCary, James y otros.(2000) Sexualidad humana. México D.F.: Editorial El Manual Moderno.

Rogers, Carl.(1980) El poder de la persona. México D.F.: Editorial El Manual Moderno S.A.

Segal, Jeanne. (1997) Su inteligencia emocional. Barcelona: Grijalbo. 